\title{
Calculation of the Effective Gravitational Charge using the Newton- Schrödinger Equations
}

\author{
Yu.N. Zayko* \\ Russian Presidential Academy of National Economy and Public Administration,Stolypin Volga RegionInstitute, \\ Saratov, Russia \\ *Corresponding Author: Yu.N. Zayko, Russian Presidential Academy of National Economy and Public \\ Administration, Stolypin Volga Region Institute, Saratov, Russia
}

\begin{abstract}
In the work, the Schrödinger-Newton equations are numerically investigated, which describe the interaction of neutral massive particles (neutrons) in neglecting their spin with the gravitational field surrounding them. An anti-screening effect of the gravitational charge of bare neutrons was found, what leads to an increase in the gravitational charge of the neutron at small distances (in galactic scale) while maintaining the Newtonian character of the gravitational potential. At large distances, the character of the gravitational potential differs from that of Newtonian, and the direction of the action of the gravitational force changes to the opposite. This allows one to qualitatively explain the phenomena known as dark matter and dark energy.
\end{abstract}

Keywords: Schrödinger-Newton equations, gravitational potential, dark energy, dark matter.

\section{INTRODUCTION}

The theory of gravity formulated by Newton in the 17th century and received a new interpretation at the beginning of the 20th century thanks to the works of A. Einstein, currently faces serious problems, of which three main ones can be identified:

- The problem of quantization of gravity

- The problem of the hidden mass, known as dark matter

- The problem of the behavior of the law of gravity at very large distances which is associated with the fact of accelerated expansion of the Universe which was confirmed by experiment.

This paper is mainly devoted to the discussion of the 1st problem. It is also shown that the two other problems are connected with the solution of the first.

The development of physics is marked by the interpenetration of the ideas underlying the description of electromagnetic and gravitational phenomena. As R. Feynman mentioned in his lectures [1], the similarity of descriptions of electromagnetic and gravitational interactions and the success of the Einstein geometric approach to the description of gravity makes many researchers look for ways to geometrize electrodynamics. On the other hand, the successes of quantum electrodynamics (QED) contributed to attempts to transfer its methods to gravity in order to create its quantum version. Neither first no other path, as we know, led to success.

The reason for the failure of the geometrization of electrodynamics is that due to the different chargeto-mass ratio of charged particles it is impossible to build a single geometric picture of electromagnetic interaction. The transfer of QED methods to gravity meant the transfer, first of all, of the methods of perturbation theory with its inherent problems including the presence of divergences. And if in the framework of QED these problems were overcome using the renormalization procedure, the quantum theory of gravity thus constructed turned out to be non-renormalizable. At the same time, it is not clear what the reason for the failure is - whether it is inherent in the nature of gravity or in the methods of its research.

Nevertheless, the use of a formal analogy between electromagnetic and gravitational phenomena (EMG analogy) can be useful, firstly, because it allows reducing the calculations, and, secondly, as shown below, avoids the problems associated with divergences and detect similarity some physical consequences when considering these different in essence phenomena. 
A different method for studying gravity based on a no perturbative approach is proposed below. It is based on the search for solutions of the Schrödinger-Newton equations (SN), which describe the effect of the gravitational field created by a massive particle on the source of the field.

The SN equations have been investigated many times before, however, for other purposes. R. Penrose used them to substantiate his ideas about the gravitational mechanism of the reduction of the wave function during the measurement procedure [2, 3]. In [4], spherically symmetric solutions of SN equations were numerically studied; their stability was studied in [5].

The purpose of this work is to apply the SN equations to cosmology problems, including the description of screening and anti-screening of a gravitational charge due to vacuum polarization, as well as deviations of the form of the gravitational potential from Newtonian at galactic distances.

The issues of calculating the amendments to the law of Newton were the subject of numerous publications $[6,7,8]$. A common feature of all the works was the consideration of gravity as field theory and the use of its methods. In these papers, the first approximation corrections were calculated, taking into account relativistic and quantum effects. The presence of anti-screening due to vacuum polarization at distances of the order of a Planck length was noted. Note that in the present work the speed of light $\mathrm{c} \rightarrow \infty$ which corresponds to the zero Planck length, i.e. the results of this work are of a macroscopic nature, in contrast to those mentioned.

\section{SCHRÖDINGER-NEWTON EQUATIONS}

We first consider the Schrödinger-Poisson (SP) equation, which describes the electron-photon interaction. Its detailed derivation, given in [9], we omit

$$
\begin{aligned}
& \frac{1}{r^{2}} \frac{\partial}{\partial r}\left(r^{2} \frac{\partial \varphi}{\partial r}\right)+\frac{2 m}{\hbar}(\omega-e \Phi) \varphi=0 \\
& \frac{1}{r^{2}} \frac{\partial}{\partial r}\left(r^{2} \frac{\partial \Phi}{\partial r}\right)=-4 \pi e|\varphi|^{2}
\end{aligned}
$$

Equations (1) describe a Dirac electron interacting with a field of virtual photons that carries a charge $-e$, neglecting its spin and two positron components of a bispinor. They are obtained by expanding the exact Dirac-Maxwell equations in powers of $1 / c^{2}(c-$ is light speed in a vacuum) [10]. In addition, consideration is limited to spherically symmetric solutions for the scalar potential of the EM field $\Phi$ and electronic components with different spin projections of the form $\varphi=\left(\begin{array}{l}\varphi_{1} \\ \varphi_{2}\end{array}\right)$, $\varphi(r, t)=\varphi(r) \exp (-i \varepsilon t / \hbar), \omega=\varepsilon-m c^{2}, m-$ is the mass of a bare electron, $\varepsilon$ - energy, $\hbar$ Planck constant, $|\varphi|^{2}=\left|\varphi_{1}\right|^{2}+\left|\varphi_{2}\right|^{2}$. The term containing the time derivative of $\Phi$ is omitted due to the fact that the size of the electron-photon interaction region $\Delta r$ is small and the lag effect $\sim(\Delta r / c)^{2}$ can be neglected [9].

It is convenient to present the system (1) in a dimensionless form for the variable $\chi=r \varphi$

$$
\eta_{\rho \rho}+2[v-U] \eta=0
$$

$\rho^{2} U_{\rho \rho}+2 \rho U=-4 \pi|\eta|^{2}$

$\eta=\frac{\hbar}{e \sqrt{m}} \chi, U=\frac{\hbar^{2}}{e^{3} m} \Phi, v=\frac{\hbar^{2}}{e^{4} m} \omega, \rho=\frac{e^{2} m}{\hbar^{2}} r$

The results of a numerical study of equations (2) are given in [9], where it was shown, in particular, that the use of a non-perturbative approach avoids divergences when calculating the value of the effective charge of a dressed electron.

Using the EMG analogy, we immediately obtain from equations (2) of the Schrödinger-Newton equation. The ground for the EMG analogy is the similarity of the Coulomb and Newton laws

$$
F_{C}=\frac{e_{1} e_{2}}{r^{2}}, F_{N}=G \frac{m_{1} m_{2}}{r^{2}}
$$

for the absolute value of the force of interaction between two charges $e_{1}$ and $e_{2}$ and two masses $m_{1}$ and $m_{2}$ separated by the distance $r$ each other, $G-$ is the constant of gravity. According to (3) one can 
introduce the "gravitational charge" $m \sqrt{G}^{1}$, which allows immediately to write the SchrödingerNewton equation

$$
\begin{aligned}
& \eta_{\rho \rho}+2[v-U] \eta=0 \\
& \rho^{2} U_{\rho \rho}+2 \rho U=4 \pi|\eta|^{2} \\
& \eta=\frac{\hbar}{\sqrt{G m^{3}}} \chi, U=\frac{\hbar^{2}}{\sqrt{G^{3}} m^{4}} \Phi, v=\frac{\hbar^{2}}{G^{2} m^{5}} \omega, \rho=\frac{G m^{3}}{\hbar^{2}} r
\end{aligned}
$$

where the sign of the right side of the second equation is reversed compared with (2). These equations coincide up to the notation with the SN equations in the papers [2 - 5].

In addition to stating the external similarity of equations (4) and (2), we should formulate a physical problem for equations (4) . To do this, we consider an electrically neutral particle of mass $m$ having a spin $1 / 2$ (neutron) described in our approximation by a two-component spinor $\eta$ and surrounded by a gravitational field with potential $\Phi$. This problem differs from the electron problem in the field of virtual photons, described by equations (2) only by scale transformations and an obvious change in the sign of the charge density of virtual particles. A more complete discussion of the physical sense of equations (4) is given below.

Let's make an assessment of the scale. Unit of length $\hbar^{2} / G m^{3}$ for the neutron mass has an order of value $0,2 \cdot 10^{8}$ light years, what consists about $0,2 \cdot 10^{3}$ the size of an average galaxy ${ }^{2}$ and about $2,5 \cdot 10^{-4}$ the size of the Universe. We will need these values below to interpret the solutions obtained.

\section{NUMERICAL STUDY OF EQUATIONS}

In the study of equations (4), we neglected the spinor structure $\eta$. In Fig. 1, 2 show the results of a numerical study of the equations (4).
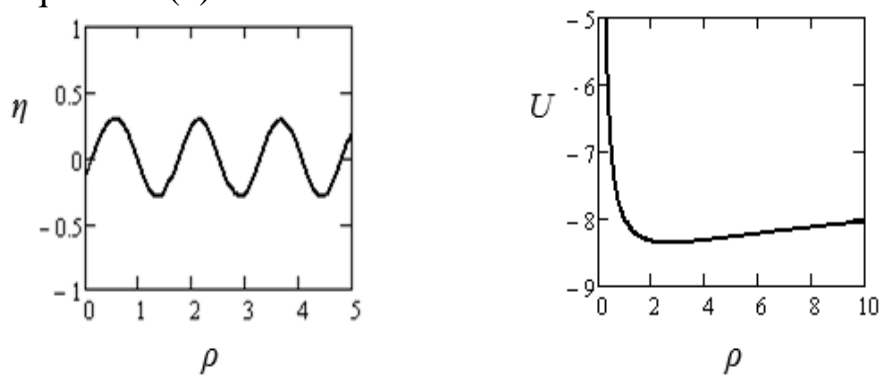

Fig1.The results of the numerical study of the equations (4) for $v=0$, i.e. the energy is measured from this level. Boundary values are set in the left turning point: $\eta=0.01, \eta=1, U=0, U^{\prime}=-70$.
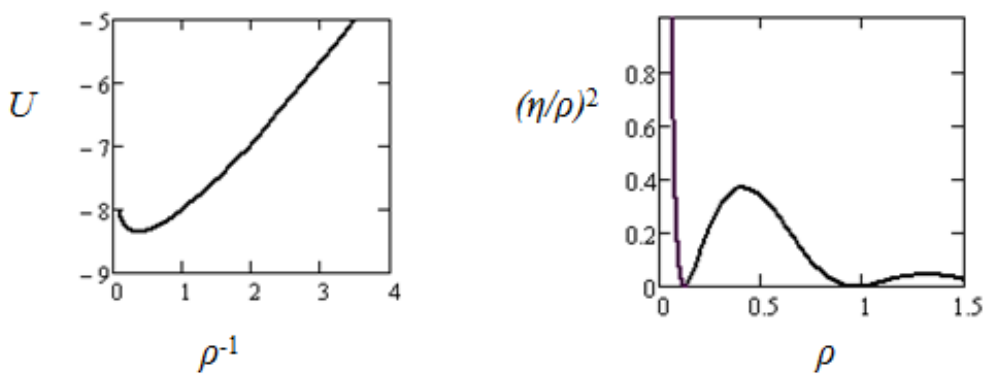

Fig2. Left: the dependence of $U\left(\rho^{-1}\right)$. Liner section (large $\left.\rho^{-1}\right)$ corresponds to the screened Newton interaction at small distances. Right: the screening gravitational charge density $(\eta / \rho)^{2}$.

Based on the above results, we can conclude that the bare neutron is surrounded by a cloud of virtual particles that affect the effective charge of the neutron and the effective law of gravitational interaction described by the potential $U$. At short distances (on a galactic scale), the potential is

\footnotetext{
${ }^{1}$ The presence of the root suggests that there are gravitational charges of different signs

${ }^{2}$ The order of magnitude of the gravitational analog of the Bohr radius for a neutron [1]
} 
approximately Newtonian ${ }^{3}: U \sim Z / \rho$. The effective gravitational charge of the neutron equals $Z=d U / d\left(\rho^{-1}\right)$ in the region of linear dependence $U\left(\rho^{-1}\right)$ i.e. for $\rho<0,5$ (Fig. 2, left) and for the data shown in Fig. 1, 2 approximately equals 1,374. At large distances approaching the size of the Universe, the potential $U$ differs from Newtonian and, moreover, the direction of the gravitational force determined by the derivative $d U / d \rho$ is reversed.

\section{DISCUSSION}

First of all, let's say a few words about the nature of the work. The work belongs to the field of numerical experiment; therefore, the choice of analysis method and the use of data are of particular importance. In the numerical integration of equations (4), the Runge-Kutta method was used with a constant step. The integration domain $(0, \mathrm{~b})$ was divided into two subregions $(a, b)$ and $(0, a)$ where $a$ is the turning point for the potential $U(\rho): U(a)=v$. Since the boundary conditions for the chosen integration procedure should be specified on the left boundary of the interval, integration over the interval $(0, a)$ was replaced by integration over the mirror interval $(-a, 0)$, which allows us to make the symmetry of equations (4) ${ }^{4}$. Boundary conditions for $\eta$ and $U$ in the points $a$ and $-a$ coincide, and for $\eta^{\prime}$ and $U^{\prime}$ differs by the sign. The choice of the magnitude of $a$ does not change the general character of the solutions but determines their interpretation. In Fig. 1, 2 presents the results for $a=1.4$. An increase in $a$ leads to an increase in the effective charge $Z>1$. Critical for these boundary values is the value $a=1.2$ - for smaller values of $a$ the charge $Z<1$ what corresponds to the usual screening, which is difficult to explain from a physical point of view. The values of $Z>1$ correspond to antiscreening and have a completely transparent explanation.

It is necessary to dwell on the problem of choosing boundary values. If equations (4) are investigated for a neutron, then the quantity $a$ should have the order of magnitude of its own size (Compton wavelength), i.e. $a \sim 10^{-15} \mathrm{~m}$ or in the dimensionless form in selected units $\sim 10^{-38}$. This imposes impracticable accuracy requirements to the calculations ${ }^{5}$. Therefore, one should choose other ways to solve the problem.

In the present work, the boundary values were chosen in the range that allows numerical calculations. Then, by scale transformations, the results can be extrapolated to the real range of values. The magnitude of specific boundary values used in the calculations will not affect the result, only the order of their magnitude is important.

For anti-screening, the shielding character is the exact opposite of electrodynamics. If in electrodynamics, screening leads to a decrease in the absolute value of the effective charge of a dressed electron compared to a bare electron, the gravitational interaction, on the contrary, increases the effective charge, i.e. has the character of anti-screening. The physical reason for the polarization of vacuum by the presence of mass is apparently the production of particle-antiparticle pairs by the gravitational field of a bare mass [11].

Let us discuss possible ways of reducing the boundary values to a physically reasonable interval.

To investigate the range of real boundary values allows the symmetry of equations (4) with respect to scale transformations. It is easy to show that by changing the scale of the distance in $\lambda$ times, i.e. introducing the scale transformation $\rho=\lambda \rho^{\prime}$, we convert other values in accordance with the formulas $U=\lambda^{-2} U^{\prime}, \eta=\lambda^{-1} \eta^{\prime}, U_{\rho}=\lambda^{-3} U_{\rho^{\prime}}^{\prime}, \eta_{\rho}=\lambda^{-2} \eta_{\rho^{\prime}}^{\prime}$. The magnitude of the shielded charge is converted as $Z=\lambda^{-1} Z^{\prime}$. In other words, if the solution of equations (4) was obtained for the supergalactic distance, then reducing the scale of the distance to an acceptable one, we, in accordance with the formulas, will achieve reduction of the remaining scales to values in the real range, while the shielded charge must remain greater than one.

The physical reason affecting the decrease in spatial scale can be the formation of neutron complexes containing $N \gg>1$ particles due to the neutron correlation, similar to the pairing of nucleons within neutron stars [12]. For such complexes, the mass of which is equal to $m N$ (if we neglect the binding

${ }^{3}$ Strictly speaking, this is not quite the case. The linear regression equation for the potential has the form $U(x)=0.082 x^{-1}-8.892+0.838 x+0.062 x^{2}, x=1 / \rho$

${ }^{4}$ Existing integration procedures allowing setting boundary conditions in the middle of an interval do not provide the necessary accuracy.

${ }^{5}$ In QED, this problem does not arise, since the use of atomic units provides an acceptable order of magnitudes of variables appearing in the calculations. 
energy in comparison with the rest energy $m c^{2}$ ) the value of its own length scale will decrease by $N^{3}$ times compared with the neutron one, i.e. $\lambda \sim N^{3}$, and for $N \sim 10 \div 100$ will be of the order or less than the size of the galaxy. At the same time, if the shielding effect for a bare neutron occurred in the ranges ${ }^{6} \Delta U, \Delta \eta$,..then for the neutron complex the corresponding ranges will be $N^{6} \Delta U, N^{3} \Delta \eta, .$. However, the issue of the formation of such complexes is currently insufficiently studied.

Analysis of cosmological observations should help in deciding the choice of real boundary values, but it is beyond the scope of this work. So far, we can state the qualitative agreement of the results with known facts - the seeming presence of additional hidden mass (dark matter) in galaxies and the acceleration of the expansion of the universe by changing the gravitational attraction by repulsion at distances of the order of the size of the universe (dark energy problem).

\section{CONCLUSION}

The work is devoted to a numerical study of the Schrödinger-Newton equations, describing the gravitational field of neutral massive particles (neutrons or neutron complexes) while neglecting their spin. It has been shown that under certain conditions, the anti-screening of the gravitational charge of bare particles occurs, leading to an increase in their effective gravitational charge at small (on galactic scales) distances. At the same time, the potential of the gravitational field preserves the Newtonian form. At large distances of the order of the size of the universe, the form of the gravitational potential differs from that of Newton, and the direction of action of the gravitational force changes to the opposite. This allows us to qualitatively explain the phenomena known as dark matter and dark energy outside the frame of $\Lambda \mathrm{CDM}$ theory.

\section{REFERENCES}

[1] R. P. Feynman, F.P. Morinigo, W.G. Wagner, Feynman Lectures on Gravitation, Ed. By B. Hartfield,1995,Addison-Wesley Publishing Co.

[2] R.Penrose, On gravity's role in quantum state reduction, Gen.Rel.Grav. 28 (1996) 581-600.

[3] R.Penrose, Quantum computation, entanglement and state reduction, Phil.Trans.R.Soc. (London) A 356 (1998) 1927.

[4] I. M. Moroz, R. Penrose, and P. Tod, Spherically-symmetric solutions of the Schrödinger-Newton equations, Class. Quantum Grav. 15 (1998) 2733-2742.

[5] R. Harrison, I. Moroz and K. P. Tod, A numerical study of the Schrödinger -Newton equations, Nonlinearity 16, (2003), pp. 101-122.

[6] A. Marunovic, T. Prokopec, Antiscreening in perturbative quantum gravity and resolving the Newtonian singularity, arXiv:1209.4779v2 [hep-th] 30 Aug 2013.

[7] J. F. Donoghue, Leading Quantum Correction to the Newtonian Potential, arXiv:gr-qc/9310024v2 25 Mar 1994.

[8] A. Marunovic, T. Prokopec, Time transients in the quantum corrected Newtonian potential induced by a massless nonminimally coupled scalar field, Phys. Rev. D 83 (2011) 104039 [arXiv:gr-qc/1101.5059].

[9] Yu.N. Zayko, On the Perturbation Theory in Quantum Electrodynamics Using the Wave Functions of the Dressed States, International Journal of Applied Mathematics and Theoretical Physics, V. 2, \# 4, Oct. 2016, P.: 28-30

[10] V. B. Berestetskii, E. M. Lifshitz, L. P. Pitaevskii (1971). Relativistic Quantum Theory. Vol. 4 (1st ed.). Pergamon Press.

[11] Ya. B. Zel'dovich, A. A. Starobinskii, Particle Production and Vacuum Polarization in an Anisotropic Gravitational Field, Soviet Phys. JETP, Vol. 34, No.6, pp. 1159-1412.

[12] A. Gezerlis, C. J. Pethick, A. Schwenk, Pairing and superfluidity of nucleons in neutron stars, arXiv:1406.6109v2 [nucl-th] 13 Apr 2015.

Citation:Yu.N. Zayko, (2019).Calculation of the Effective Gravitational Charge using the NewtonSchrödinger Equations. International Journal of Scientific and Innovative Mathematical Research (IJSIMR), 7(6), pp.17-21. http://dx.doi.org/10.20431/2347-3142.0706003

Copyright:@ 2019 Authors, this is an open-access article distributed under the terms of the Creative Commons Attribution License, which permits unrestricted use, distribution, and reproduction in any medium, provided the original author and source are credited.

${ }^{6}$ Although the calculations were performed for different (close) boundary values of $U, \eta, \ldots$, the evaluation of the ranges in which the screening effect exists did not perform 